\title{
Antibiotic prophylaxis in transarterial therapy of hepatocellular carcinoma: A meta-analysis
}

\author{
Jun Wang MD, Xiao Dong He MD, You Cheng Zhang MD PhD
}

\begin{abstract}
J Wang, XD He, YC Zhang. Antibiotic prophylaxis in transarterial therapy of hepatocellular carcinoma: A meta-analysis. Can J Gastroenterol 2012;26(2):85-91.
\end{abstract}

BACKGROUND: The use of prophylactic antibiotics against postprocedure infection in patients undergoing transarterial therapy for hepatocellular carcinoma is controversial.

AIM: To compare the effects of prophylactic antibiotic treatment and no prophylactic antibiotic treatment on infectious complications following transarterial procedures.

METHODS: Clinical trials fulfilling predefined selection criteria were identified by searching several bibliographic databases; a meta-analysis was performed where appropriate.

RESULTS: Four trials of inadequate quality consisting of 210 patients were included in the analysis. Only one case of possible postprocedure infection in each group was reported. The rate of patients developing fever (RR 0.91 [95\% CI 0.61 to 1.35]), changes in peripheral white blood cell count or serum C-reactive protein levels, and the mean length of hospital stay (mean difference 0.20 [95\% CI 0.75 to 1.14]) showed no significant intergroup differences between antibiotic and no antibiotic treatment. Furthermore, the results of the present study indicated that the incidence of bacteremia, septicemia, sepsis or hepatic abscess after transarterial therapy was rare.

CONCLUSION: Antibiotic prophylaxis in patients undergoing transarterial therapy for hepatocellular carcinoma may not be routinely necessary. However, a more judicious use of antibiotics is recommended for patients who are at an increased risk of infection. Nevertheless, prospective trials on a larger scale are clearly needed.

Key Words: Antibiotic prophylaxis; Hepatocellular carcinoma; Infection; Meta-analysis; Transarterial therapy

$\mathrm{W}$ orldwide, hepatocellular carcinoma (HCC) is the sixth most common malignancy, with an estimated mortality rate of $9.2 \%$ in 2008 (1). Transarterial therapy, including transarterial embolization (TAE), and transarterial chemoembolization (TACE) and transarterial chemotherapy (TAC), have been used increasingly in patients with HCC since it was demonstrated to improve survival, either as the sole treatment or in conjunction with surgery or local ablative therapies (2-4). The rationale for using the above interventional procedures to treat HCC is based on the difference in anatomical configuration between tumour tissue and normal hepatic parenchyma, by which devascularization of tumour and/or a high concentration of chemotherapeutic agents delivered to cancer cells can be achieved.

Among the various modalities of transarterial therapy, both TAE and TACE aim to occlude hepatic arterial flow with embolizing agents, while TACE procedures are preceded by the administration of anticancer agents. TAC, which is also known as transarterial infusion chemotherapy or transarterial oily chemoembolization, consists only of hepatic arterial infusion with chemotherapeutic agents, usually uses lipiodol as a vehicle and is not designed to achieve arterial occlusion

\section{La prophylaxie antibiotique dans la thérapie transartérielle du carcinome hépatocellulaire : une méta-analyse}

HISTORIQUE : Le recours à une prophylaxie antibiotique contre l'infection après une thérapie transartérielle du carcinome hépatocellulaire est controversé.

OBJECTIF : Comparer les effets d'une antibiothérapie prophylactique et de l'absence d'un tel traitement sur les complications infectieuses après des interventions transartérielles.

MÉTHODOLOGIE: Les auteurs ont repéré des essais cliniquesrespectant certains critères prédéfinis au moyen de recherches dans plusieurs bases de données bibliographiques. Ils ont procédé à une méta-analyse, au besoin.

RÉSULTATS : Quatre essais de qualité insuffisante, composés de 210 patients, ont été inclus dans l'analyse. Un seul cas d'infection possible après l'intervention a été signalé. Le taux de patients faisant de la fièvre (RR 0,91 [95 \% IC 0,61 à 1,35]), présentant des modifications de la numération des globules blancs périphériques ou des taux de protéines $\mathrm{C}$ réactive, de même que la durée d'hospitalisation moyenne (différence moyenne 0,20 [95\% IC 0,75 à 1,14]) n'ont révélé aucune différence intergroupe significative entre le traitement antibiotique et l'absence de traitement antibiotique. De plus, les résultats de la présente étude indiquent que l'incidence de bactériémie, de septicémie, de sepsie ou d'abcès hépatique était rare après une thérapie transartérielle.

CONCLUSION : La prophylaxie antibiotique chez les patients qui subissent une thérapie transartérielle en raison d'un carcinome hépatocellulaire n'est peut-être pas systématiquement nécessaire. Cependant, une utilisation plus judicieuse des antibiotiques est recommandée pour les patients qui sont plus vulnérables à l'infection. De toute évidence, des essais prospectifs à plus grande échelle s’imposent.

(5). All of these techniques have been widely performed for more than 20 years and are generally well tolerated. However, these procedures were reported to be accompanied by several infectious complications such as bacteremia and septicemia - even sepsis and hepatic abscess especially when performed in patients with HCC, indicating that the prophylactic use of antibiotics may be necessary for the prevention of such infections (6-8). Some groups suggested that broad-spectrum antibiotic(s) should be routinely given to patients with HCC before any invasive procedure because these patients often had hepatic functional damage and were at a high risk of developing bacterial infections (9-11). Indeed, HCC patients usually present with cirrhosis, which may favour immunosuppression. On the other hand, immune function in these patients may be further impaired if they are exposed to anticancer agents (12). Clinically, although a uniform consensus on the regimens against postprocedure infection has not yet been reached, three to seven days of antibiotic coverage for Gram-negative enteric organisms was recommended for patients who underwent TACE (13). However, postprocedure infection occurs, even in patients who previously received antibiotic prophylaxis. In a study involving 157 patients

Department of General Surgery, Lanzhou University Second Hospital, Lanzhou, Gansu, China

Correspondence: Dr You Cheng Zhang, Department of General Surgery, Lanzhou University Second Hospital, Lanzhou 730030, Gansu, China.

Telephone 86-931-8942287, fax 86-931-8458109, e-mail zhangychphd@yahoo.cn

Receieved for publication May 11, 2011. Accepted June 29, 2011 
who were treated with cefazolin and metronidazole before and after TACE, hepatic abscess was reported to develop in $4.5 \%$ of the population (14). Therefore, antibiotic prophylaxis appears to be necessary for patients undergoing transarterial therapy for HCC. A recent prospective trial that evaluated the efficacy of two different antibiotic regimens (15) further emphasized the importance of antibiotics in prophylaxis against infection after transarterial therapy for HCC.

In contrast, other studies revealed that the incidence of immediate or long-term infection events following transarterial procedures were minor and, therefore, suggested that prophylactic antibiotic use may not be necessary $(7,16-19)$. This was proposed based on the awareness that the negative effects of indiscriminate antibiotic use may result in increased bacterial resistance and medical costs. Furthermore, trials have also indicated that administration of prophylactic antibiotics may decrease the overall prevalence of infectious complications after TAE, but may not decrease the incidence of liver abscesses $(9,20)$. Taking into account that there have been only a few reports on the rate of infections complicating transarterial therapy, the use of prophylactic antibiotics against postprocedure infection is, therefore, controversial. Using curently available clinical trial evidence, the aim of the present meta-analysis was to assess the effects of prophylactic antibiotic therapy versus no prophylactic antibiotic treatment with regard to infectious complications in patients undergoing transarterial therapy for HCC.

\section{METHODS}

\section{Literature search}

An electronic search was performed using the key words "hepatocellular carcinoma", "embolization", "chemoembolization" and "antibiotic prophylaxis", which included searches in the Cochrane Library (Cochrane Central Register of Controlled Clinical Trials), Medline (via PubMed), Embase and Chinese biomedicine literature database (CBM). Both subject and text terms were used, and were combined according to a predesigned search strategy. The database searches were not restricted to English language publications or data. However, only clinical trials were considered for inclusion in the present study. In addition, the reference lists of the retrieved articles were manually screened for other potentially relevant studies.

\section{Selection criteria}

Trials evaluating the use of prophylactic antibiotic treatment in patients with HCC undergoing transarterial therapy were eligible to be included if they compared any antibiotic or combination of antibiotics with either placebo or no treatment. However, because the aim of the current meta-analysis was to investigate the necessity of prophylactic antibiotic treatment in patients undergoing transarterial therapy for HCC, studies that compared the effects of different antibiotics or different regimens (dosages, routes or durations of administration) of one antibiotic were not included. Both randomized controlled trials (RCTs) and non-RCTs were considered for the present study; however, studies lacking control groups were excluded. Trials containing mixed indications were retained if the results for each indication could be extracted separately.

\section{Outcomes measurement}

Primary outcomes: Any proven systemic infection such as bacteremia, septicemia and sepsis (confirmed with positive blood culture), hepatic abscess (confirmed with radiological features) and other clinical indications of infection, however defined by individual studies.

Secondary outcomes: Suspected infection or clinical signs of infection such as fever, elevated white blood cell (WBC) count or C-reactive protein (CRP) level, and duration of hospital stay.

\section{Data extraction and analysis}

Two authors (J Wang and YC Zhang) independently assessed the inclusion eligibility of the potential studies identified in the search according to the criteria outlined above, with any disagreement resolved by discussion. A standardized data collection form was used to extract relevant information and outcomes data from the included studies. Details regarding trial design, participants, transarterial techniques, antibiotic regimens and outcome measures were retrieved and abstracted. Important patient baseline characteristics from each trial were also reviewed.

All outcomes data were extracted on a worksheet, and were crosschecked for accuracy before being combined for analysis by two of the authors (J Wang and XD He). A meta-analysis was applied based on the absence of considerable clinical heterogeneity among the included trials and was performed with RevMan software version 5.1.1 (provided by the Cochrane Collaboration). A descriptive summary of the results was presented when the outcome data from different studies could not be combined. For dichotomous variables, such as the number of patients developing infection, the treatment effects were reported as RR with $95 \% \mathrm{CI}$, whereas mean difference (MD) and its corresponding $95 \%$ CI were used to express continuous variables such as duration of hospital stay. A fixed-effect model was used for pooling the results unless there was significant heterogeneity, in which case a random-effect model was applied.

Heterogeneity was assessed by visual inspection of forest plots and the $\chi^{2}$ test, with statistical significance set at $\mathrm{P}>0.1$. The $\mathrm{I}^{2}$ statistic was used to estimate total variation across studies that was due to heterogeneity and expressed as a percentage: $<25 \%$ was considered to be low level heterogeneity; $25 \%$ to $50 \%$ as moderate level and $>50 \%$ as high level (21). If moderate levels of heterogeneity (ie, $\mathrm{I}^{2}>50 \%$ ) were encountered, possible sources of heterogeneity were explored. A sensitivity analysis was performed to examine the potential risk bias in the overall results of pooled analysis, (eg, results of non-RCTs were excluded from the analysis for these of RCTs).

\section{Quality assessment}

Methodological quality was assessed independently by two authors (J Wang and XD He) using the criteria outlined in version 5 of the Cochrane Handbook for Systematic Reviews of Interventions (22). Four items assessing components of internal validity of trials, including method of randomization, allocation concealment, blinding and reporting of an intention-to-treat (ITT) analysis, were used to evaluate study quality, but only for RCTs. Each item was scored as 'yes' for low risk of bias, 'unclear' for either lack of information or uncertainty over the potential for bias, and 'no' for high. Trials with a retrospective design were considered to be of inadequate quality; however, this assessment did not determine its inclusion or exclusion.

\section{Search results}

\section{RESULTS}

Among 191 nonduplicated articles retrieved by the electronic searches, 188 were excluded mainly due to their clear irrelevance because they were nonclinical trials or were uncontrolled (Figure 1); however, one study identified from the reference list of a published article was found to meet the criteria for inclusion (18). Finally, four studies consisting of 210 patients from Europe, Asia and North America were included in the current meta-analysis (16-19).

\section{Characteristics of the included studies}

General information: All of these four studies were clinical trials conducted between 1992 and 2007. The general information is summarized in Table 1. The outcomes of interest were reported by all of the trials, in most of which both confirmed and suspected infection events were studied. Factors associated with postprocedure fever were also analyzed (16-17). In the trial by Wang et al (19), however, the only outcome that was considered to be clinically relevant for which data were presented was rate of infection. Overall, patients were generally well matched between the treatment and control groups for demographics, etiology of HCC, hepatic function and type of transarterial therapy within each study (16-18) (Table 2). However, one trial did not describe the characteristics of the included populations (19). 
Types of transarterial modalities: Of the four trials, two $(17,19)$ used TACE to treat HCC and one (16) used TAE. HCC patients in the remaining trial were treated with TAE, TACE or transarterial Yttrium-90 (18). Two trials $(16,18)$ detailed the transarterial technique that was used for HCC, while the procedure was not described in the other two $(17,19)$. One trial $(16)$ included 75 TAE procedures performed in 61 HCC patients, but provided all outcomes data referring to the $75 \mathrm{TAE}$ procedures. Therefore, in the current meta-analysis, every patient was considered to have undergone a TAE treatment; these data were analyzed accordingly.

Antibiotic protocols: Among the included trials, intravenous (IV) cephalosporin alone (29 of 112) or cephalosporin combined with metronidazole ( 83 of 112 ) was the antibiotic(s) mostly used before transarterial treatment, with reported treatment durations of $0.5 \mathrm{~h}$ to longer than $48 \mathrm{~h}(16,18-19)$. Also, ciprofloxacin was administered either as an initial (15 of 112) or a maintenance therapy (data were not provided) against postprocedure infection (17). However, the antibiotic regimens varied among studies. Castells et al (16) used a cross-over policy of antibiotics for a suspected bacterial infection, in which patients allocated to the no treatment group were given the regimen used in the antibiotic group, and those in the antibiotic group were considered for an empirically alternative antibiotic protocol when infection was suspected. Plentz et al (17) administered a combination of IV metronidazole with ciprofloxacin or cephalosporin to patients in the antibiotic group for the first three days after TACE. The IV regimen was subsequently changed to an oral combination of metronidazole with ciprofloxacin if febrile and

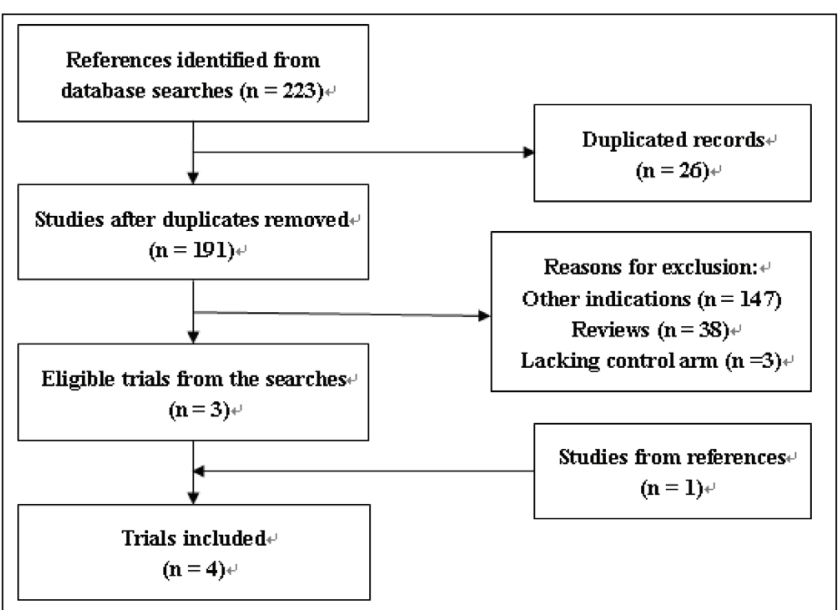

Figure 1) Flow diagram of the literature searches

symptomatic signs of infection disappeared. Either IV or oral regimen was continued for an additional seven days. Thus, every patient in the study group was treated for 10 days with two antibiotics. In the trial by Wang et al (19), monotherapy with cefazolin was used before patients underwent TACE. The remaining trial (18) only reported the antibiotic agents used before and/or after the transcatheter procedure; however, detailed information regarding the regimens was not given.

\section{TABLE 1}

General information of the included studies

\begin{tabular}{lllll}
\hline $\begin{array}{l}\text { Author } \\
\text { (reference), year }\end{array}$ & Country & $\begin{array}{l}\text { Included } \\
\text { populations }\end{array}$ & $\begin{array}{l}\text { Transarterial } \\
\text { techniques }\end{array}$ & $\begin{array}{l}\text { Antibiotic } \\
\text { regimen }\end{array}$ \\
\hline Castells et al & Spain & Patients with HCC, & TAE, with embolizing agents of Study group ( $\mathrm{n=37):} \mathrm{IV}$ & $\begin{array}{l}\text { Primary } \\
\text { end points }\end{array}$
\end{tabular}

(16), $1995 \quad$ confirmed by tumour
Wang et al (19), China 2005

Patients with primary liver cancer

Plentz et al (17), Germany 2005

Shelgikar et al USA (18), 2009

Patients with histological or serological evidence biopsy and/or elevated AFP, and radiological findings gelatin cube $(1 \mathrm{~mm} \times 1 \mathrm{~mm})$ alone or gelatin and steel coil. The use of lipiodol was not described
Patients who had previously not received any treatment for nonresectable HCC. The diagnosis of $\mathrm{HCC}$ was based on elevated AFP level and/or fine-needle liver biopsy

\section{Only stated as TACE,} information on the procedure was not provided cefotaxime $2 \mathrm{~g} / 6 \mathrm{~h}+$ metronidazole $500 \mathrm{mg} / 8 \mathrm{~h}$ before TAE and for at least 48 h. Control group $(n=38)$ : no antibiotic treatment

Study group $(n=29)$ : IV cefazolin $2 \mathrm{~g}$ before TACE for $0.5 \mathrm{~h}$. Control group $(n=31)$ : no antibiotic treatment

Only stated as TACE, the use Study group ( $n=15)$ : IV of anticancer drugs, embolizing agents and lipiodol were not described ciprofloxacin $200 \mathrm{mg}+$ metronidazole $500 \mathrm{mg}$ on the morning of TACE. IV cephalosporin (not specified) twice a day + metronidazole $500 \mathrm{mg}$ three times a day for the following 2 days. Seven days of IV cephalosporin + metronidazole $500 \mathrm{mg}$, or oral ciprofloxacin $250 \mathrm{mg}+$ metronidazole $500 \mathrm{mg}$. Control group ( $n=15)$ : no antibiotic treatment. of $\mathrm{HCC}$

Study group $(n=31)$ : one
TAE, TACE, or Yttrium-90. The embolizing agent was polyvinyl alcohol particles and a cisplatin-based regimen mixed with ethiodol was used for chemoembolization dose of cefazolin and metronidazole were given before the transarterial procedure (8 patients), or pre- and postprocedure (23 patients). Control group ( $n=28)$ : no antibiotic treatment
2 months after Postprocedure TACE infection

5 weeks after Adverse effects discharge

$\begin{array}{lc}\text { Median } & \text { Infectious } \\ 28 \text { months } & \text { complications }\end{array}$

chemoembolization; TAE Transarterial embolization 
TABLE 2

Baseline patient characteristics in the included studies

\begin{tabular}{|c|c|c|c|c|c|c|c|c|c|}
\hline \multirow[b]{2}{*}{$\begin{array}{l}\text { Author } \\
\text { (reference), year }\end{array}$} & \multirow[b]{2}{*}{$\begin{array}{c}\text { Patients } \\
\text { included, } \mathbf{n}\end{array}$} & \multirow[b]{2}{*}{$\begin{array}{l}\text { Age, } \\
\text { years }\end{array}$} & \multirow[b]{2}{*}{ Male,\% } & \multirow{2}{*}{$\begin{array}{c}\text { Etiology } \\
\text { HBVIHCVI } \\
\text { other, } n\end{array}$} & \multicolumn{2}{|c|}{ Characteristics of HCC } & \multirow{2}{*}{$\begin{array}{c}\text { Liver function } \\
\text { Child-Pugh } \\
\text { class }(A / B, n)\end{array}$} & \multicolumn{2}{|c|}{ Laboratory parameters } \\
\hline & & & & & $\begin{array}{c}\text { Tumour lesions } \\
\text { (solitary/multiple), } \mathrm{n}\end{array}$ & AFP, ng/L & & $\begin{array}{l}\text { WBC count, } \\
\times 10^{9} / L^{*}\end{array}$ & $\begin{array}{l}\text { CRP, } \\
\mathrm{mg} / \mathrm{L}^{*}\end{array}$ \\
\hline \multicolumn{10}{|c|}{ Castells et al (16), 1995} \\
\hline Antibiotic arm & 37 & $60 \pm 9 *$ & 86 & $26^{\dagger} / 11$ & $8 / 29$ & $22 / 15^{\ddagger}$ & $31 / 6$ & $5.2 \pm 2.1$ & NR \\
\hline Control arm & 38 & $61 \pm 8^{*}$ & 79 & $35^{\dagger} / 3$ & $4 / 34$ & $17 / 11^{\ddagger}$ & $30 / 8$ & $4.8 \pm 2.1$ & \\
\hline \multicolumn{10}{|c|}{ Wang et al (19), 2005} \\
\hline Antibiotic arm & 31 & NR & NR & NR & NR & NR & NR & NR & NR \\
\hline Control arm & 29 & & & & & & & & \\
\hline \multicolumn{10}{|c|}{ Plentz et al (17), 2005} \\
\hline Antibiotic arm & 15 & $62.2^{\S}$ & 87 & $3 / 4 / 8$ & $6 / 9$ & $996 \pm 1467^{*}$ & $15 / 0$ & $7.3 \pm 2.5$ & $11.6 \pm 12.4$ \\
\hline Control arm & 15 & $65.2^{\S}$ & 87 & $2 / 3 / 10$ & $5 / 10$ & $595 \pm 1134^{*}$ & $15 / 0$ & $6.8 \pm 2.3$ & $7.6 \pm 7.9$ \\
\hline \multicolumn{10}{|c|}{ Shelgikar et al (18), 2009} \\
\hline Antibiotic arm & 31 & $56^{\S}$ & 78 & $3 / 30 / 26$ & NR & $18^{\S}$ & NR & NR & NR \\
\hline Control arm & 28 & & & & & & & & \\
\hline
\end{tabular}

${ }^{*}$ Data presented as mean $\pm S D ;{ }^{\dagger}$ Hepatitis including hepatitis $B$ virus (HBV) and hepatitis C virus (HCV); ${ }^{\ddagger}$ Number of patients with serum alpha-fetoprotein (AFP) level $\leq 100 \mathrm{ng} / \mathrm{mL} />10 \mathrm{ng} / \mathrm{mL}$; ${ }^{\S}$ Data presented as median. CRP C-reactive protein; HCC Hepatocellular carcinoma; NR Not reported; WBC White blood cell

TABLE 3

Quality assessment of randomized controlled trials

\begin{tabular}{lllll}
\hline $\begin{array}{l}\text { First author } \\
\text { (reference) }\end{array}$ & Randomization & $\begin{array}{l}\text { Allocation } \\
\text { concealment }\end{array}$ & Blinding & ITT analysis \\
\hline Castells (16) & Unclear & Unclear & No & Yes \\
Wang (19) & Unclear & No & No & Yes \\
Plentz (17) & Unclear & No & No & Yes \\
\hline
\end{tabular}

ITT Intention to treat

Methodology quality assessment

Among the four trials, three $(16,17,19)$ were retrieved as RCTs, while the other (18) was a retrospective controlled study. One trial was a Chinese publication with an English abstract (19), while another (17) was a correspondence. The methodological quality assessment for the included RCTs are presented in Table 3. All of the three RCTs $(16,17,19)$ stated that patients were randomly assigned to the treatment and control options, but failed to describe the precise method of randomization within the studies. Although allocation concealment was achieved using sealed envelopes in the trial by Castells et al (16), it was unclear whether the envelope was opaque (22). However, this procedure was not reported in either of the two other trials $(17,19)$. Furthermore, the three trials $(16,17,19)$ appeared to have no blinding of intervention or outcome assessment, and data were presented on a perprotocol basis. In addition, there were no dropouts or loss to follow-up reported in the trials (16-19).

\section{Effects of interventions}

Bacteremia, septicemia and sepsis: Transarterial procedures carry risks for several types of blood infections such as bacteremia, septicemia and sepsis, which were clinically evidenced with positive blood culture results. Because TAE, TACE and TAC involve a percutaneous arterial approach, bacteria can be introduced exogenously from skin puncture. On the other hand, tumour necrosis and normal liver parenchyma induced by embolization and/or chemotherapy can become a source of infection, especially through bacterial reflux via the biliary system from the intestine (23). Hence, infection occurs through a different route of bacterial transmission. The most common types of pathogenic organisms were Staphylococcus, Streptococcus, Klebsiella and Escherichia coli, while infection with fungi was rare $(7,9,24)$. Without antibiotic prophylaxis, bacteremia occurred in $3.6 \%$ of patients undergoing hepatic arteriography (HA) and $1.1 \%$ of those undergoing TAE. Septicemia occurred in $1.1 \%$ of pateints who underwent TACE, and sepsis occurred in $1.7 \%$ of patients who underwent TAE $(6,7)$.
None of the blood infections was detected among patients in the included three trials, regardless of whether they were treated with or without antibiotics (16-18). Castells et al (16) used biological cultures from blood, urine and ascitic fluid for the examination of bacterial infections, whereas the diagnosis of blood infections was not described by the other two studies $(17,18)$. In the trial by Wang et al (19), one patient in each group developed a hyperpyrexia with acute symptoms of toxicity after TACE, indicating that an early episode of septicemia would be expected. However, it was unknown from the study whether the author diagnosed the infection using the results of biological fluid cultures. The one case of this possible infection that occurred despite the use of antibiotic prophylaxis was reported in a patient who underwent repeated TACE performed through the same skin puncture, whereas one patient in the control group was elderly with compromised hepatic functional reserve. Nevertheless, given the limited information on the criteria regarding blood infections within the individual trials, together with the reported zero incidence rate of any of the infection outcomes, the results could not be presented as a pooled analysis.

\section{Hepatic abscess}

Hepatic abscess is a severe complication associated with transarterial procedures. The typical symptoms indicating hepatic abscess include fever, chills and right upper quadrant pain, which usually occur on the first to sixth day after the procedure (25). Radiologically, hepatic abscesses appear as areas of hypodensity on computed tomography (CT) scan and hypoechogenicity on ultrasonogram. However, the confirmed diagnosis of hepatic abscess is based on ultrasound-guided fine-needle aspiration of the suspected liver lesions. Both Grampositive and Gram-negative bacteria can lead to cases of pyogenic liver abscess. Gram-positive aerobes reportedly accounted for $60 \%$ of pathogenic organisms in TAE-related liver abscess $(9,26)$. When antibiotic prophylaxis was not applied, the incidence of this complication among patients with hepatic tumours after TAC was $0.2 \%$, and among those after TAE was $0.2 \%$ to $1.1 \%(6,8,26,27)$. In a recent study that reviewed 11 articles (28), hepatic or splenic abscesses were estimated to occur in a median of $1.3 \%$ of patients.

Only in one of the four trials evaluated in the present study was the outcome of hepatic abscess reported using CT findings (18). Neither the number of patients developing hepatic abscess nor the methods for diagnosis of hepatic abscess were stated in the other three trials $(16,17,19)$. However, no patients in the trial were found to develop hepatic abscess during a median follow-up duration of 28 months (18). 


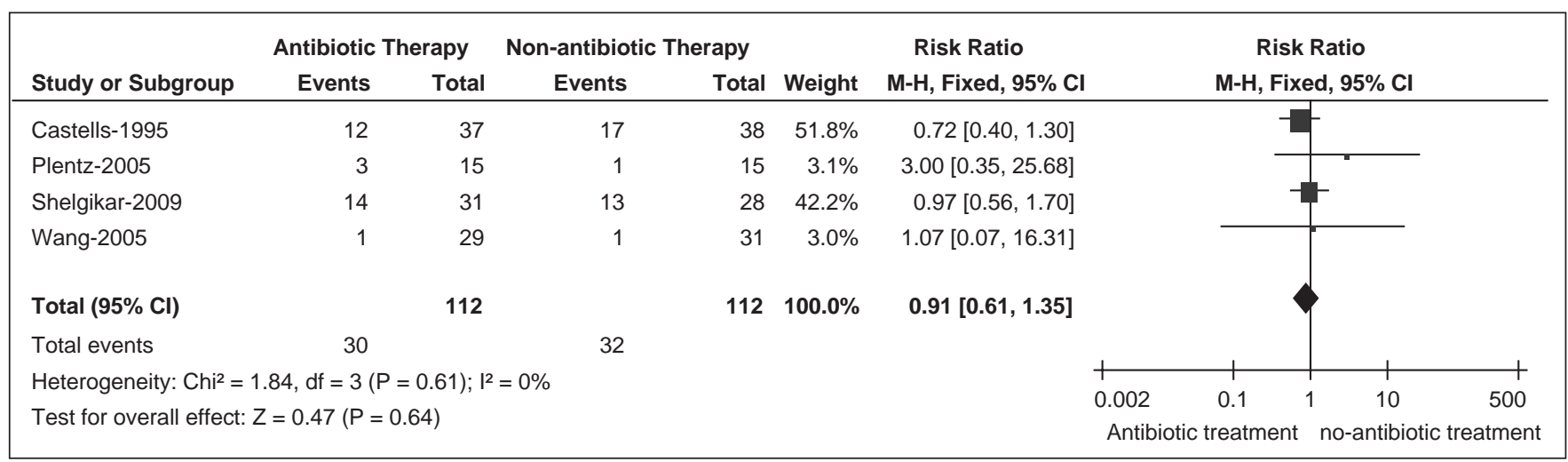

Figure 2) Fever. Pooled analysis for all trials

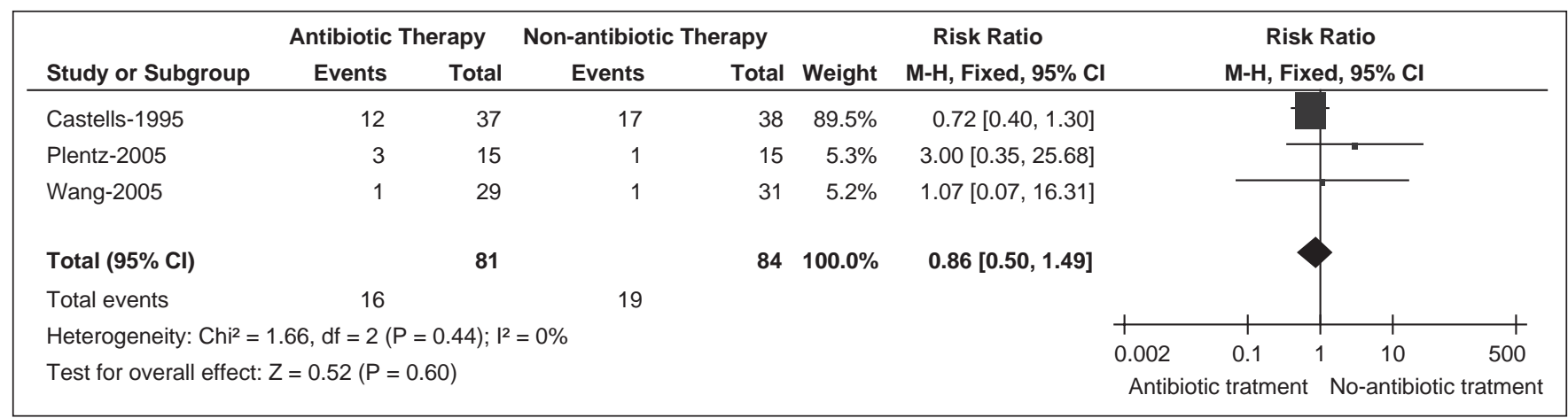

Figure 3) Fever. Sensitivity analysis for randomized controlled trials

\section{Fever}

Clinically, the appearance of fever after a transarterial procedure is considered to be a symptomatic sign of secondary infection. However, it is also known that fever, which is one of the typical features of postembolization syndrome that occurs in $60 \%$ to $80 \%$ of patients undergoing transarterial procedures, represents a clinical marker of tumour necrosis and, thus, of a favourable response to treatment $(7,16,17)$. Most cases of fever reportedly occurred on the day of transarterial therapy or immediately after transarterial procedures, and were usually self-limited to within three to four days (28). Other postembolization symptoms, such as nausea, vomiting, abdominal pain and increase in peripheral WBC count or CRP level, may also be observed during the period of fever. In clinical settings, although there has been no clear evidence for the use of antibiotics in the treatment of postprocedure syndrome, patients may have already received prophylactic antibiotics before these symptoms were apparent.

The number of patients with fever after transarterial therapy for HCC was reported by all of the included trials, in two of which only patients with fever $>38^{\circ} \mathrm{C}$ were recorded $(16,17)$. Meta-analysis of the outcome showed that there was no significant difference between prophylactic antibiotic and no antibiotic treatment (RR 0.91 [95\% CI 0.61 to 1.35$]$ ), and this result was stable in prospective data (RR 0.86 [95\% CI 0.50 to 1.49 ]), in which the non-RCT study (18) was excluded (Figures 2 and 3). Moreover, no statistical heterogeneity was seen in either of the comparisons. Also, there were no significant differences in the mean duration of the fever and its maximal value between the two groups, as reported in the trial by Castells et al (16). Most fevers peaked on postprocedure day 1 to 3, without elevation of WBC or CRP levels, and the overall incidence of patients developing fever across the trials was $26.8 \%$ for the antibiotic group and $28.6 \%$ for the no antibiotic group. However, none of these patients demonstrated evidenced of a bacterial infection (16-18).

\section{Elevated WBC or CRP}

Determination of the levels of two inflammatory markers - WBC and CRP - is widely performed because increased levels are the common markers of infection. Some studies considered the appearance of fever with concomitantly increased WBC and/or CRP levels as a sign of suspected infection and thereby an indication for using antibiotics, while in others, the symptoms were demonstrated to be the result of tumour necrosis induced by transarterial therapy (15-17).

Changes in serum levels of CRP and/or WBC in all patients, or in those with fever were reported in three trials (16-18). Due to the variations in criteria and weighting of the two parameters across the trials, pooled analyses for these outcomes were limited. However, there was no significant intergroup differences with regard to these two inflammatory markers at any of the assessed time points (16-18). No patients with elevated WBC and/or CRP levels demonstrated evidence of a bacterial infection.

\section{Duration of hospital stay}

Of the four trials, three reported the duration of hospital stay (in days) of the included patients using a statistical mean and SD (16-18). Combining the results of two RCTs $(16,17)$ showed no significant difference between the antibiotic and no antibiotic groups (MD 0.20 [95\% CI -0.75 to 1.14$]$ ]). Heterogeneity was not observed in this comparison (ie, $\mathrm{I}^{2}=0 \%$ ). Also, no difference was found in the duration of hospital stay in the remaining trial (18) (Figure 4).

\section{DISCUSSION}

The current meta-analysis demonstrated that of 210 enrolled patients from four clinical trials, only one in each group experienced a possible postprocedure infectious complication. The rate of patients developing fever, changes in serum WBC and CRP levels, and the mean length of hospital stay showed no significant intergroup differences between antibiotic and no antibiotic treatment. Furthermore, the results of the present study suggest that the incidence of bacteremia, septicemia, sepsis or hepatic abscess after transarterial therapy is rare. Therefore, the use of prophylactic antibiotics in patients undergoing transarterial therapy for HCC may not be routinely necessary.

Several series suggested that patients with biliary tract disease, such as ischemia, injury, congestion or inflammation, or those who 


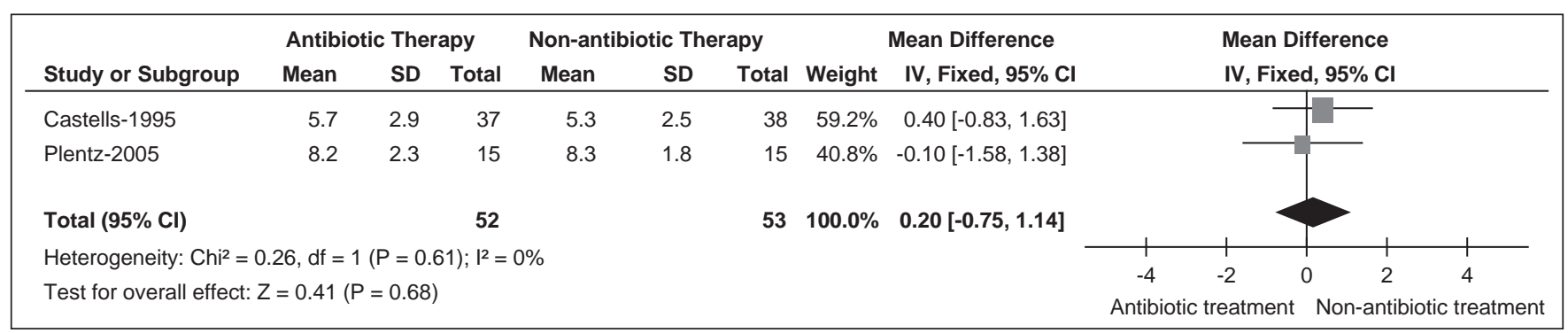

Figure 4) Duration of hospital stay

underwent previous biliary reconstruction surgery or enterobiliary anastomosis, were at high risk of developing infectious complications following transarterial therapy for $\operatorname{HCC}(7,25,26,29)$. In a small RCT by Geschwind et al (24), the authors compared the effects of two antibiotic regimens for prophylaxis against hepatic abscesses after TACE for HCC in patients with a history of biliary reconstructive surgery. The results showed that none of the patients assigned to receive IV tazobactam/piperacillin developed hepatic abscess after TACE, whereas the complication occurred in those whom cephalexin was given. In the present analysis, however, none of the included patients were found to have biliary disease or a history of biliary surgery. Nevertheless, more aggressive antibiotics with coverage against Grampositive, Gram-negative aerobic and anaerobic organisms may be indicated for these patients $(24,30)$. Also, postprocedure infections have been shown to be associated with advanced age, larger tumour nodules, presence of portal vein thrombosis or compromised hepatic function, and previous gastrectomy $(6,7,9,19,25,26)$. Due to the lack of prospective data, however, it has been unclear whether patients with these factors are at an increased risk of infectious complications after transarterial procedures. Nevertheless, the relationship between the above factors and postprocedure infections requires further investigation, and more judicious use of antibiotic prophylaxis for these patients is recommended.

Fever, elevated WBC and CRP were mostly indicated because the postembolization syndromes resulted from tumour necrosis and may not be considered as routine signs of bacterial infection. Because it is unclear whether tumour necrosis represents a sensitive predictor of treatment response or an adverse response to transarterial therapy, the appearance of postembolization syndrome should be treated with caution (31-34). On the other hand, because fever frequently occurred among patients undergoing transarterial procedures, it is difficult to differentiate between fever due to tumour necrosis and fever due to secondary infection in clinical settings. However, an infection should be suspected when unexpectedly high and prolonged fever, and sudden onset of high fever with chills are encountered after transarterial procedures. Again, the exclusive effects of infectious complications can not be detected only by changes in WBC and CRP levels. Therefore, a surveillance strategy for the ascertainment of infection events should still be based on the results of bacterial cultures, and future studies should specify the criteria for the diagnosis of such infections.

The current meta-analysis had several limitations. First, it included only four trials with a limited number of subjects $(n=30$ to $n=75)$, which may be largely responsible for the indiscriminately negative results of any of the infection-related outcomes. Therefore, more prospective trials of sufficient sample size assessing the effects of antibiotic use in patients undergoing transarterial therapy for HCC are clearly needed. Because the incidence rate of confirmed bacteremia after transarterial procedures for HCC without antibiotic prophylaxis was $1.1 \%$ to $3.6 \%$, and that of liver abscess was $0.2 \%$ to $4.5 \%$, a worthwhile study to determine the possible effects of prophylaxis on such infections might need to enrol 1000 patients $(6-8,14,26)$.

Second, the impact of the type of transarterial procedures on infection outcomes could not be assessed separately. In a study that examined the incidence of patients undergoing HA and TAE (6), TAE was found to be more likely associated with a higher risk of developing symptomatic bacteremia than was HA ( $4 \%$ versus $0 \%$, respectively $[\mathrm{P}=0.03]$ ), suggesting that the increased frequency of the infection in the TAE group may be the result of the changes in the host induced by embolization. The possible explanation was that ischemic damage to the nontumoural liver by the occlusive effects of embolizing agents may precipitate or exacerbate liver failure, especially among cirrhotic patients with an advanced Child-Pugh class, resulting in deterioration of intrahepatic immunological defense mechanisms. Furthermore, differences may also exist between embolization and chemoembolization because local administration of chemotherapeutic agents may lead to tumour necrosis and necrosis of normal liver tissues due to toxicity and subsequent infection. Although there has been no study comparing these two procedures on the incidence rate of infections, nonsignificant differences between TACE and TAI regarding infectious complications and changes in WBC and CRP levels were found in a recent trial (15) that indicated chemoembolization and chemotherapy may also have no effects on postprocedure infection. However, all of the patients enrolled in the study were treated with antibiotics preprocedurally and, by doing so, the difference in the risk of developing infections after the procedures may have been compromised. Therefore, variations in transarterial therapy modalities may result in different prevalences of postprocedure infections; however, this requires further study.

Finally, the overall quality of the included trials was poor. Of the three RCTs, two failed to provide information on any of the items used for assessing the methodological validity of RCTs, while in the remaining one, only the method of allocation concealment was described but with an insufficient definition. Furthermore, another retrospective study was included in the meta-analysis. Thus, all of the included trials were scored as inadequate quality (22). Consequently, although meta-analyses were performed without statistical heterogeneity, combining the results of trials with methodological weakness may contribute to the risk of bias to the pooled analysis. Furthermore, none of the included trials used placebo as a control but involved 'no treatment' as a comparator. However, effects between placebo and no treatment may be different, particularly in trials with small populations (35). Given the paucity of studies focusing on antibiotic use in patients undergoing transarterial procedures for HCC, the current study provided results with the most currently available evidence. Nevertheless, more prospective data are expected.

\section{CONCLUSION}

Antibiotic prophylaxis against postprocedure infectious complications in patients undergoing transarterial therapy for HCC may not be routinely necessary. However, a more judicious use of antibiotics is recommended for patients with increased risk of infection, especially for those with concurrent biliary tract disease or a history of biliary reconstruction surgery. Nevertheless, prospective trials on a larger scale are clearly needed.

ACKNOWLEDGEMENTS: This meta-analysis was supported by a grant from Natural Science Foundation of Gansu Province, China (No. 096RJZA070). 


\section{REFERENCES}

1. Ferlay J, Shin HR, Bray F, et al. Estimates of worldwide burden of cancer in 2008: GLOBOCAN 2008. Int J Cancer 2010;127:2893-917.

2. Camma C, Schepis F, Orlando A, et al. Transarterial chemoembolization for unresectable hepatocellular carcinoma: Meta-analysis of randomized controlled trials. Radiology 2002;224:47-54.

3. Ramsey DE, Geschwind JF. Chemoembolization of hepatocellular carcinoma - what to tell the skeptics: Review and meta-analysis. Tech Vasc Interv Radiol 2002;5:122-6.

4. Marelli L, Stigliano R, Triantos C, et al. Treatment outcomes for hepatocellular carcinoma using chemoembolization in combination with other therapies. Cancer Treat Rev 2006;32:594-606.

5. Llovet J M, Bruix J. Systematic review of randomized trials for unresectable hepatocellular carcinoma: Chemoembolization improves survival. Hepatology 2003;37:429-42.

6. Chen C, Tsang YM, Hsueh PR, et al. Bacterial infections associated with hepatic arteriography and transarterial embolization for hepatocellular carcinoma: a prospective study. Clin Infect Dis 1999;29:161-6.

7. Chung JW, Park JH, Han JK, et al. Hepatic tumors: Predisposing factors for complications of transcatheter oily chemoembolization. Radiology 1996;198:33-40

8. Song SY, Chung JW, Han JK, et al. Liver abscess after transcatheter oily chemoembolization for hepatic tumors: Incidence, predisposing factors, and clinical outcome. J Vasc Interv Radiol 2001;12:313-20.

9. Reed RA, Teitelbaum GP, Daniels JR, Pentecost MJ, Katz MD. Prevalence of infection following hepatic chemoembolization with cross-linked collagen with administration of prophylactic antibiotics. J Vasc Interv Radiol 1994;5:367-71.

10. Wyke RJ. Bacterial infections complicating liver disease. Baillieres Clin Gastroenterol 1989;3:187-210.

11. Spies JB, Rosen RJ, Lebowitz AS. Antibiotic prophylaxis in vascular and interventional radiology: A rational approach. Radiology 1988;166:381-7.

12. Lu W, Li YH, He XF, et al. [Effect of dosage of anticancer agents during transcatheter arterial chemoembolization on T cell subsets in patients with hepatocellular carcinoma.] Di Yi Jun Yi Da Xue Xue Bao 2002;22:524-6.

13. Brown DB, Cardella J F, Sacks D, et al. Quality improvement guidelines for transhepatic arterial chemoembolization, embolization, and chemotherapeutic infusion for hepatic malignancy. J Vasc Interv Radiol 2006;17:225-32.

14. Ramsey DE, Kernagis LY, Soulen MC, Geschwind JF. Chemoembolization of hepatocellular carcinoma. J Vasc Interv Radiol 2002;13:S211-21.

15. Ebisutani C, Sato S, Nishi K, et al. Antibiotic prophylaxis in transcatheter treatment of hepatocellular carcinoma: An open randomized prospective study of oral versus intravenous administration. Intern Med 2010;49:1059-65.

16. Castells A, Bruix J, Ayuso C, et al. Transarterial embolization for hepatocellular carcinoma. Antibiotic prophylaxis and clinical meaning of postembolization fever. J Hepatol 1995;22:410-5.

17. Plentz RR, Lankisch TO, Bastürk M, et al. Prospective analysis of German patients with hepatocellular carcinoma undergoing transcatheter arterial chemoembolization with or without prophylactic antibiotic therapy. J Gastroenterol Hepatol 2005;20:1134-6.
18. Shelgikar CS, Loehle J, Scoggins CR, McMasters KM, Martin RC II. Empiric antibiotics for transarterial embolization in hepatocellular carcinoma: Indicated? J Surg Res 2009;151:121-4.

19. Wang JY, Chen Y, Lu W, Wei CJ. [Necessity of prophylactic antibiotics in patients with primary liver cancer undergoing transcatheter arterial chemoembolization: a prospective study.] Di Yi Jun Yi Da Xue Xue Bao 2005;25:757-8.

20. Wong E, Khardori $\mathrm{N}$, Carrasco $\mathrm{CH}$, et al. Infectious complications of hepatic artery catheterization procedures in patients with cancer. Rev Infect Dis 1991;13:583-6.

21. Higgins JP, Thompson SG, Deeks JJ, Altman DG. Measuring inconsistency in meta-analyses. BMJ 2003;327:557-60.

22. Higgins JPT, Green S, eds. Cochrane Handbook for Systematic Reviews of Interventions Version 5.1.0. The Cochrane Collaboration, 2011. <www.cochrane-handbook.org> (Accessed March 2011).

23. Hashimoto T, Mitani T, Nakamura H, et al. Fatal septic complication of transcatheter chemoembolization for hepatocellular carcinoma. Cardiovasc Intervent Radiol 1993;16:325-7.

24. Geschwind JF, Kaushik S, Ramsey DE, et al. Influence of a new prophylactic antibiotic therapy on the incidence of liver abscesses after chemoembolization treatment of liver tumors. J Vasc Interv Radiol 2002;13:1163-6.

25. de Baere T, Roche A, Amenabar JM, et al. Liver abscess formation after local treatment of liver tumors. Hepatology 1996;23:1436-40.

26. Chen C, Chen PJ, Yang PM, et al. Clinical and microbiological features of liver abscess after transarterial embolization for hepatocellular carcinoma. Am J Gastroenterol 1997;92:2257-9.

27. Sakamoto I, Aso N, Nagaoki K, et al. Complications associated with transcatheter arterial embolization for hepatic tumors. Radiographics 1998;18:605-19.

28. Marelli L, Stigliano R, Triantos C, et al. Transarterial therapy for hepatocellular carcinoma: which technique is more effective? A systematic review of cohort and randomized studies. Cardiovasc Intervent Radiol 2007;30:6-25.

29. Kim W, Clark TW, Baum RA, Soulen MC. Risk factors for liver abscess formation after hepatic chemoembolization. J Vasc Interv Radiol 2001;12:965-8.

30. Beddy P, Ryan JM. Antibiotic prophylaxis in interventional radiology - anything new? Tech Vasc Interv Radiol 2006;9:69-76.

31. Wigmore SJ, Redhead DN, Thomson BN, et al. Postchemoembolisation syndrome - tumour necrosis or hepatocyte injury? Br J Cancer 2003;89:1423-7.

32. Paye F, Farges O, Dahmane M, et al. Cytolysis following chemoembolization for hepatocellular carcinoma. Br J Surg 1999;86:176-80.

33. Takenaka K, Yoshida K, Nishizaki T, et al. Postoperative prophylactic lipiodolization reduces the intrahepatic recurrence of hepatocellular carcinoma. Am J Surg 1995;169:400-4.

34. A comparison of lipiodol chemoembolization and conservative treatment for unresectable hepatocellular carcinoma. Groupe d'Etude et de Traitement du Carcinome Hepatocellulaire. N Engl J Med 1995;332:1256-61.

35. Hrobjartsson A, Gotzsche PC. Is the placebo powerless? An analysis of clinical trials comparing placebo with no treatment. N Engl J Med 2001;344:1594-602. 


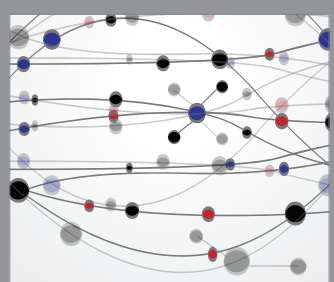

The Scientific World Journal
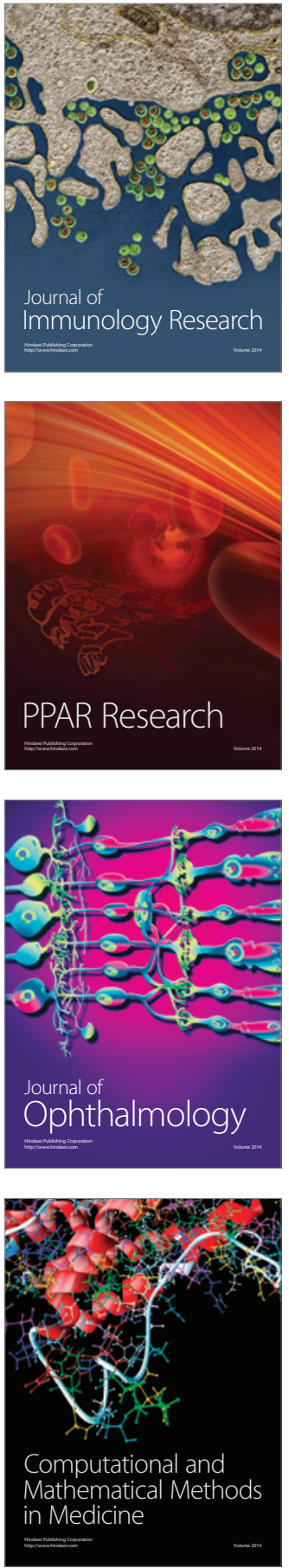

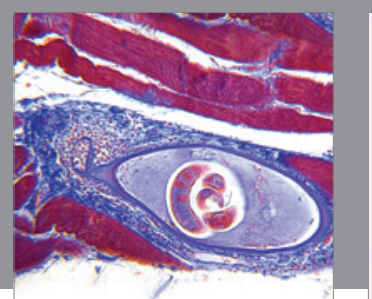

Gastroenterology Research and Practice

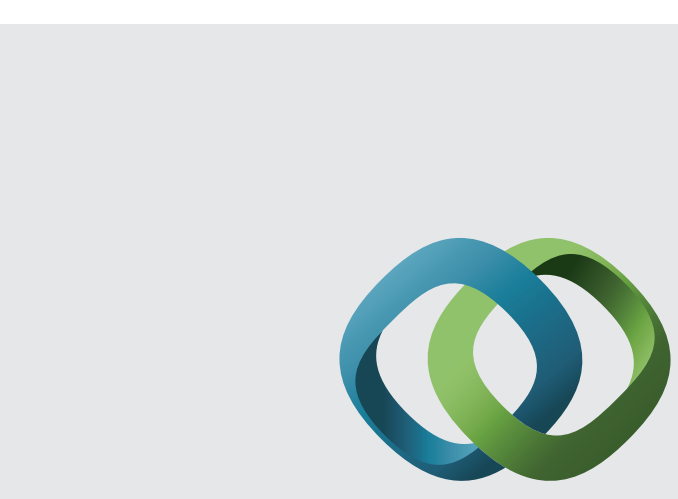

\section{Hindawi}

Submit your manuscripts at

http://www.hindawi.com
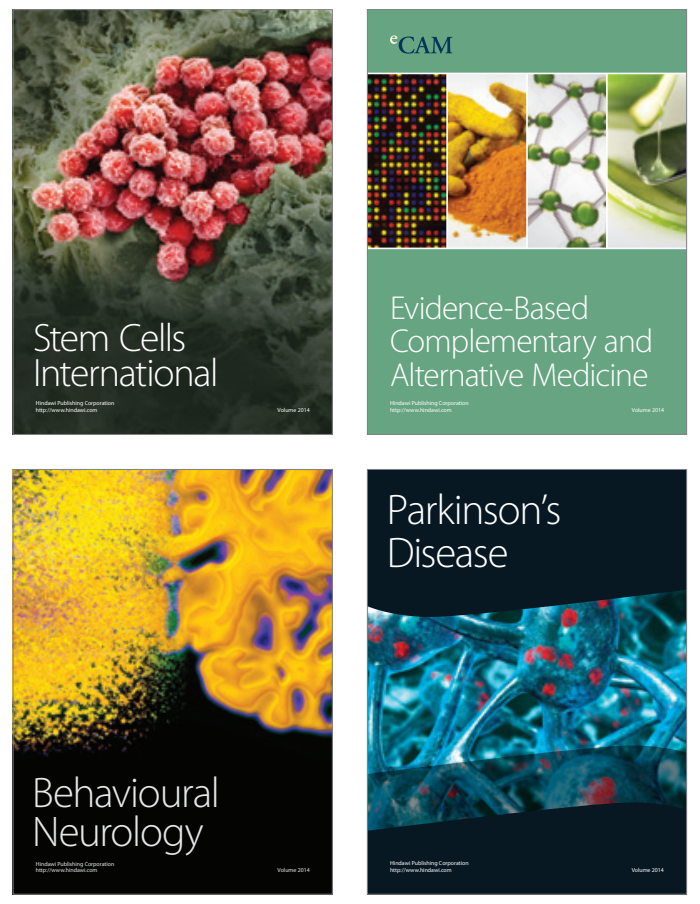
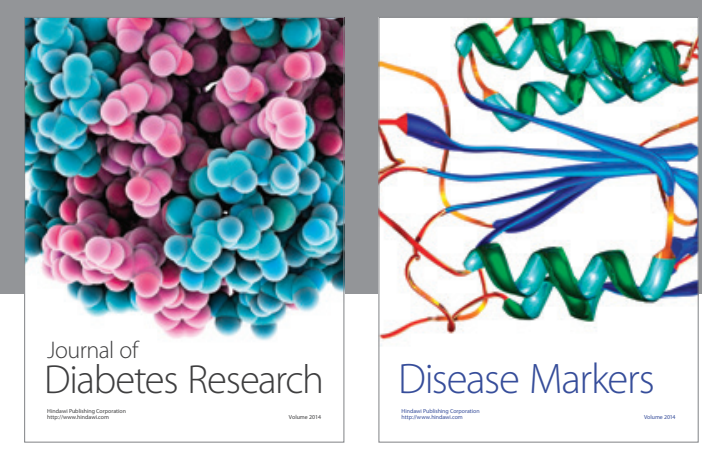

Disease Markers
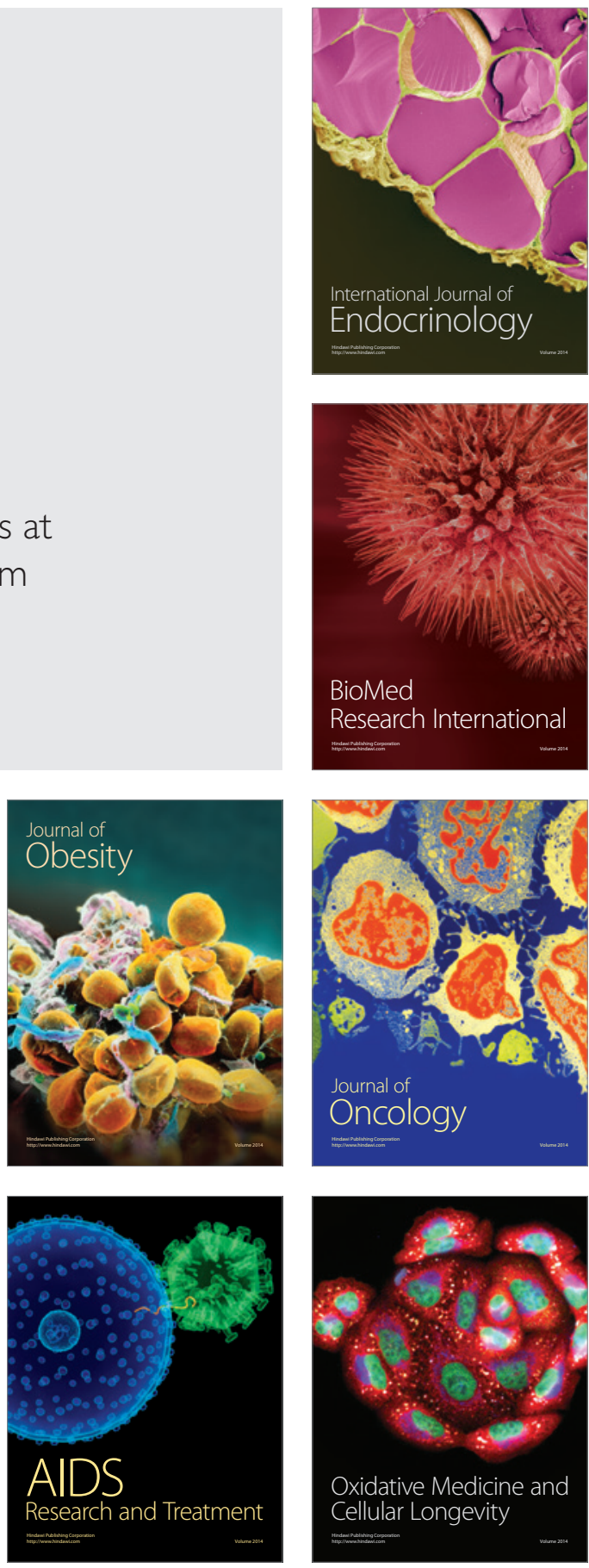